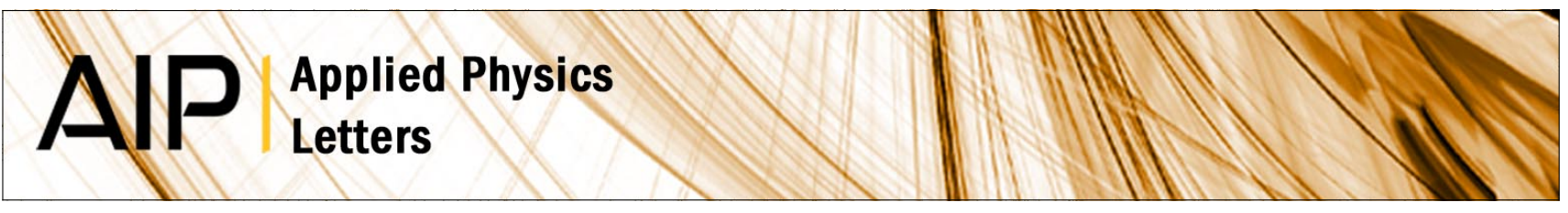

\title{
Quasimetallic silicon micromachined photonic crystals
}

B. Temelkuran, Mehmet Bayindir, E. Ozbay, J. P. Kavanaugh, M. M. Sigalas et al.

Citation: Appl. Phys. Lett. 78, 264 (2001); doi: 10.1063/1.1339256

View online: http://dx.doi.org/10.1063/1.1339256

View Table of Contents: http://apl.aip.org/resource/1/APPLAB/v78/i3

Published by the American Institute of Physics.

Additional information on Appl. Phys. Lett.

Journal Homepage: http://apl.aip.org/

Journal Information: http://apl.aip.org/about/about_the_journal

Top downloads: http://apl.aip.org/features/most_downloaded

Information for Authors: http://apl.aip.org/authors

\section{ADVERTISEMENT}

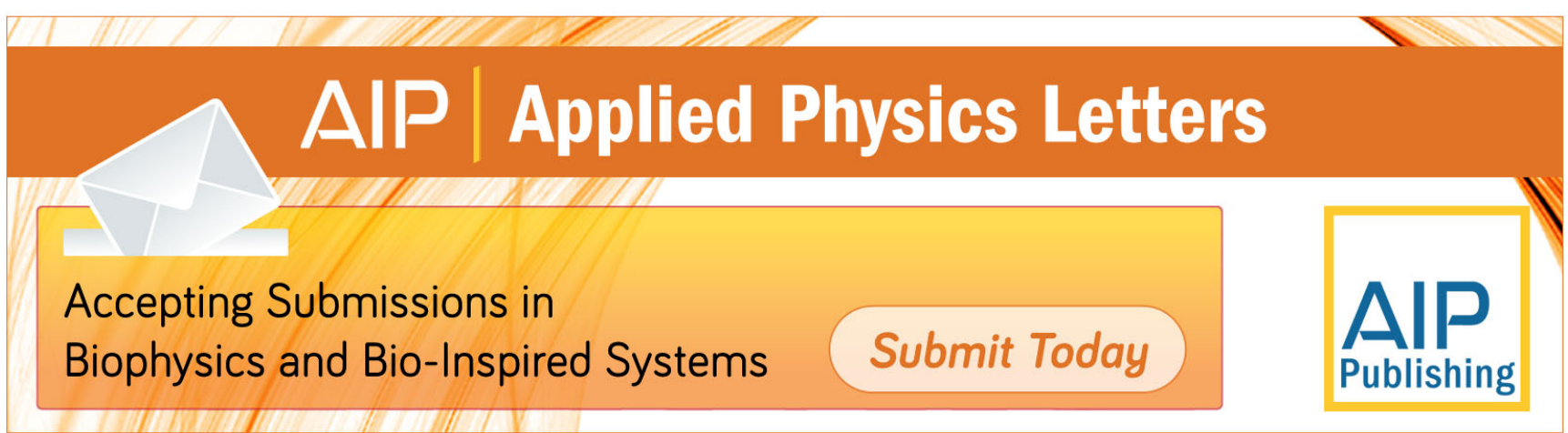




\title{
Quasimetallic silicon micromachined photonic crystals
}

\author{
B. Temelkuran, ${ }^{\text {a) }}$ Mehmet Bayindir, ${ }^{\text {b) }}$ and E. Ozbay \\ Department of Physics, Bilkent University, Bilkent 06533 Ankara, Turkey \\ J. P. Kavanaugh, M. M. Sigalas, and G. Tuttle \\ Ames Laboratory and Microelectronics Research Center, Iowa State University, Ames, Iowa 50011
}

(Received 4 May 2000; accepted for publication 9 November 2000)

\begin{abstract}
We report on fabrication of a layer-by-layer photonic crystal using highly doped silicon wafers processed by semiconductor micromachining techniques. The crystals, built using (100) silicon wafers, resulted in an upper stop band edge at $100 \mathrm{GHz}$. The transmission and defect characteristics of these structures were found to be analogous to metallic photonic crystals. We also investigated the effect of doping concentration on the defect characteristics. The experimental results agree well with predictions of the transfer matrix method simulations. (c) 2001 American Institute of Physics.

[DOI: $10.1063 / 1.1339256]$
\end{abstract}

Photonic crystals are periodic structures that can reflect electromagnetic (EM) waves in all directions within a certain frequency range. These structures can be used to control and manipulate the behavior of EM waves. ${ }^{1,2}$ Although earlier work concentrated on building these crystals with dielectric materials ${ }^{3,4}$ there are certain advantages of introducing metals to photonic crystals. ${ }^{5-11}$

Various techniques have been reported for the fabrication of dielectric layer-by-layer photonic crystals at different frequency regions, ${ }^{4,12,13}$ and recently at optical frequencies. ${ }^{14,15}$ However, limitations of the standard machining techniques used to fabricate three-dimensional (3D) metallic photonic crystals restricted experimental demonstrations and technological applications of these crystals to microwave frequencies. ${ }^{9,16}$ 3D metallic structures standing on dielectric supports operating at infrared wavelengths were also demonstrated. ${ }^{17,18}$ However, these structures do not have the advantage of a band gap extending to zero frequency due to nontouching metallic layers. The fabrication of 3D metallic photonic crystals at higher (compared to microwave) frequency regions with a complete metallicity gap extending to zero frequency is still a challenge.

In this letter, we propose a method for the fabrication of layer-by-layer photonic crystals having metallic properties using silicon micromachining techniques. The touching layers form a continuous network, in which the long wavelengths cannot penetrate the conducting mesh, and the band gap extends to zero frequency. We have previously investigated the properties of such a metallic photonic crystal with an upper band edge at $20 \mathrm{GHz}$. The method allows the fabrication of these structures at a frequency range extending from $100 \mathrm{GHz}$ to $10 \mathrm{THz}$.

The layer-by-layer photonic crystal was fabricated using highly doped silicon (100) wafers, which were $75 \mathrm{~mm}$ in diameter and $400 \mu \mathrm{m}$ thick. We predicted that, due to the low resistivity of the Si wafers (in the range of 0.0015-0.004 $\Omega \mathrm{cm}$ ), this structure would show metallic photonic crystal

a) Author to whom correspondence should be addressed; electronic mail: burak@fen.bilkent.edu.tr

${ }^{b)}$ Electronic mail: bayindir@fen.bilkent.edu.tr properties. We used anisotropic etching of silicon by aqueous potassium hydroxide $(\mathrm{KOH})$ in forming the layers of the quasimetallic photonic crystal. In the first step of the process, one side of the wafers was coated with a silicon nitride film [Fig. 1(a)], which serves as a mask during the anisotropic etching step. Next, the nitride film was patterned by conven-

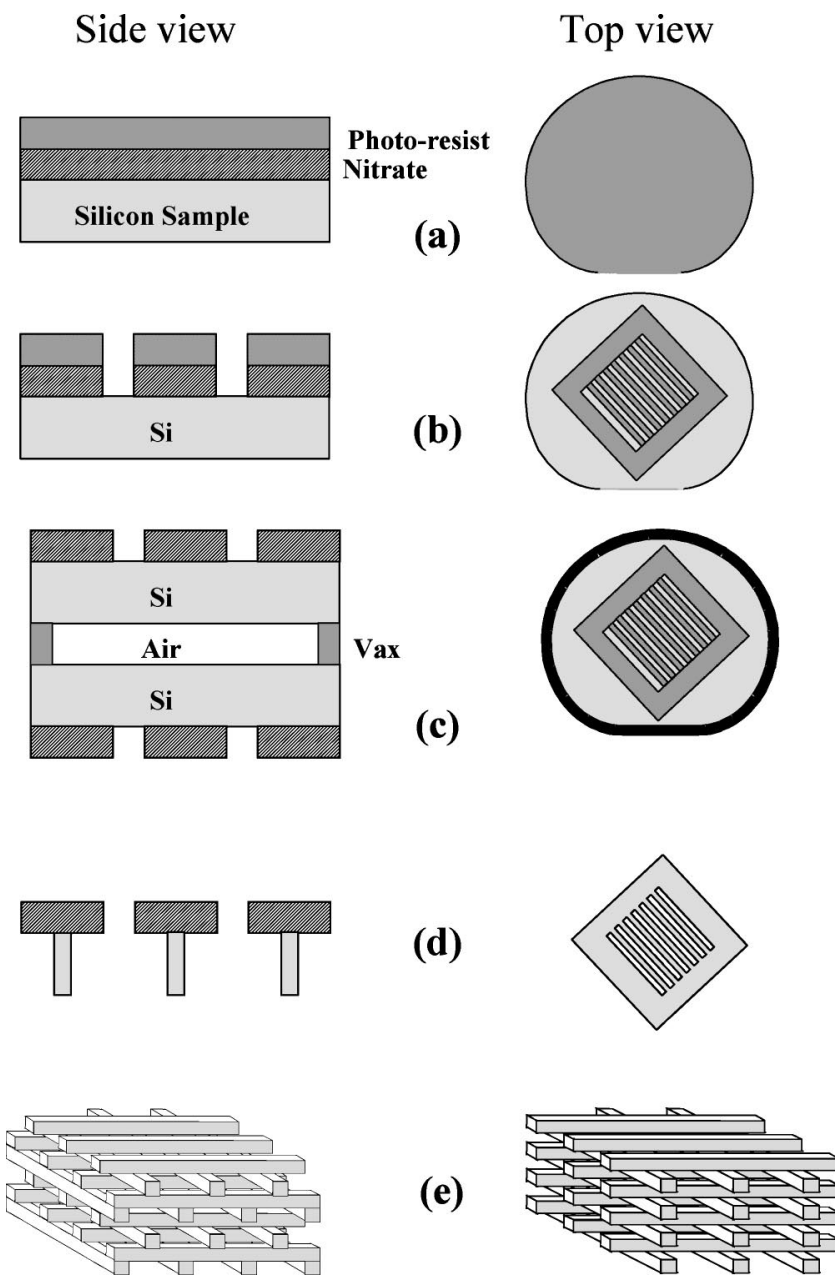

FIG. 1. (a)-(d) Micromachining process steps. (e) The micromachined wafers can be stacked to form either fct (left side) or st (right side) type photonic crystal structures. 

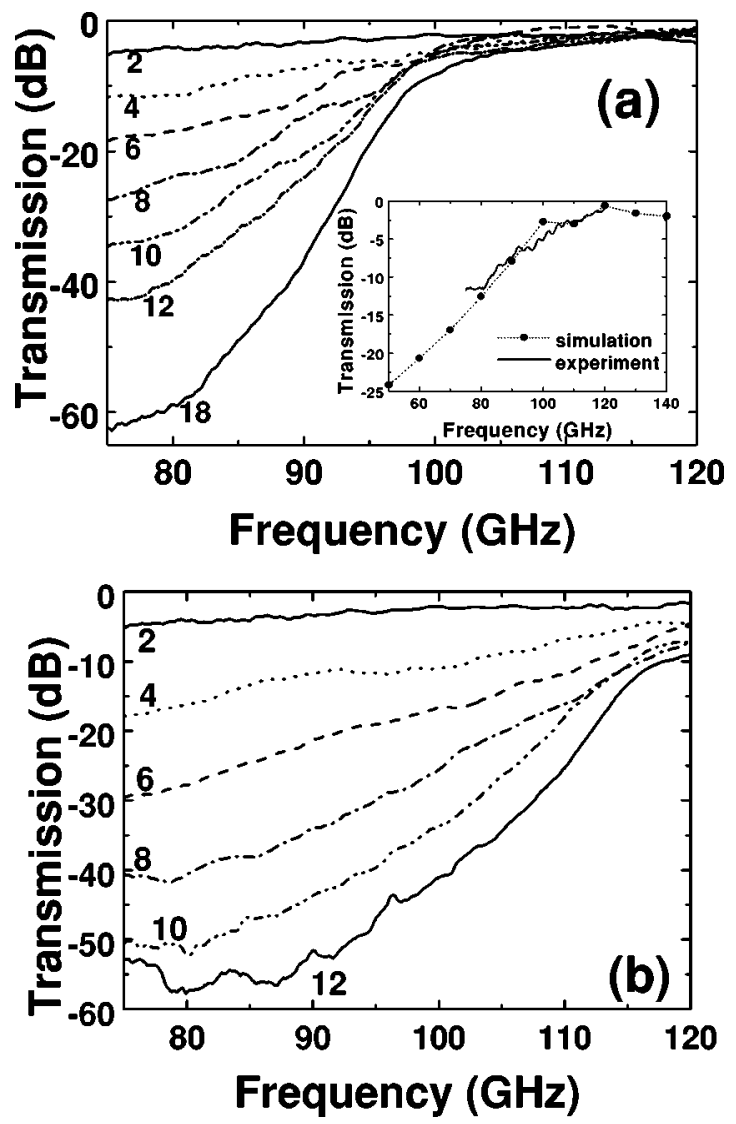

FIG. 2. Transmission characteristics of (a) st and (b) fct layer-by-layer photonic crystals (numbers indicate the number of layers). Inset: Measured (solid line) and simulated (dotted line) transmission spectra as a function of frequency for a four-layer st type crystal structure.

tional photolithography and aqueous hydrofluoric (HF) acid etching. After the silicon nitride layer is patterned [Fig. 1(b)], in order to protect back sides of the wafers, we bond each sample back to back with another patterned sample using black wax (Apiezon-W) [Fig. 1(c)]. The wafers are then dipped into an aqueous $\mathrm{KOH}$ solution. A typical etch performed in a $25 \% \mathrm{KOH}$ solution at a temperature of $55^{\circ} \mathrm{C}$ takes about $24 \mathrm{~h}$ to etch entirely through the wafers. Finally, the wax is removed by trichloroethane (TCA), so that the coupled layers bonded with wax are separated [Fig. 1(d), left side]. The remaining nitride film is removed by HF, and the samples are ready to be stacked to form photonic crystals [Fig. 1(d), right side]. The pattern consists of 19 parallel stripes (each $150 \mu \mathrm{m} \times 400 \mu \mathrm{m} \times 3 \mathrm{~cm}$ ) with center-to-center separation of $1600 \mu \mathrm{m}$. These stripe dimensions and the wafer thickness determine the upper band edge, calculated to be around $100 \mathrm{GHz}$. As shown in Fig. 1(e), the wafers (each a single layer of the quasimetallic photonic crystal) may be stacked to form a simple tetragonal (st) or a face centered tetragonal (fct) crystals.

Once fabrication is completed, transmission properties of the crystal are measured with a W-band $(75-120 \mathrm{GHz})$ measurement setup. Figure 2 shows the transmission spectra of two types of crystal structures as the number of layers is increased. The upper edge of the band gap for st crystal is located at $100 \mathrm{GHz}$ [Fig. 2(a)], and for fct crystal at $115 \mathrm{GHz}$ [Fig. 2(b)]. No lower band edge is detected within the measurable frequency range, consistent with the theory that pre-
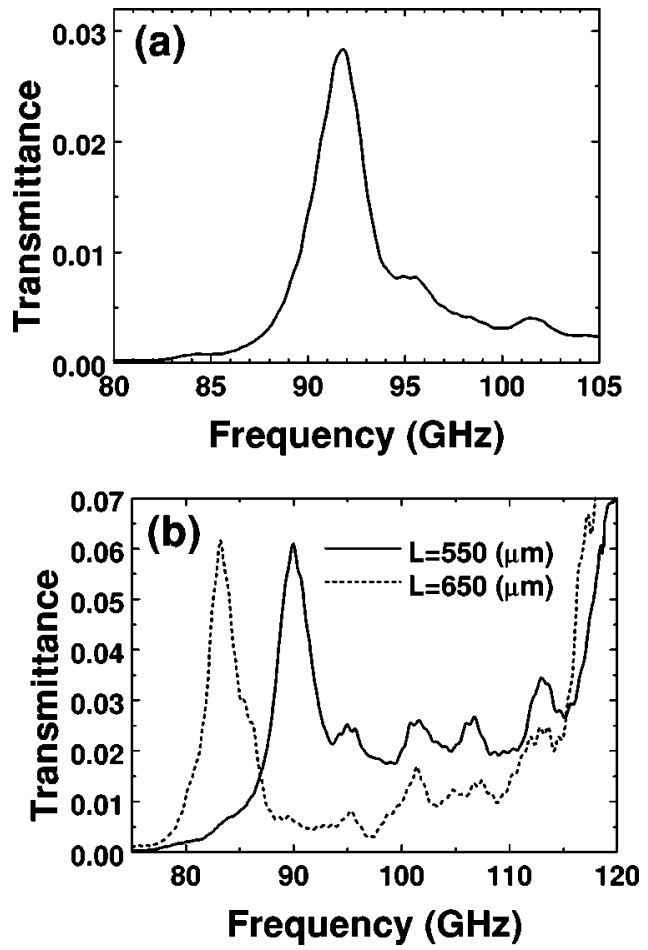

FIG. 3. (a) Transmission characteristics of the nine-layer fct type of crystal with a single rod removed defect. (b) Transmission characteristics of eightlayer fct type planar defect structures.

dicted a band gap extending down to zero frequencies. This metallicity gap verifies the prediction that our crystals are analogous to metallic photonic crystals, ${ }^{9}$ and hence can be called quasimetallic photonic crystals. At $80 \mathrm{GHz}$, the attenuation per layer is around $3.5 \mathrm{~dB}$ for the st structure and $5.3 \mathrm{~dB}$ for the fct structure. Within the metallicity gap of both of the crystals, we observe full reflection $(100 \%)$ of the EM waves.

We have simulated the transmission characteristics of these structures using the well known transfer matrix method (TMM). ${ }^{19,20}$ Using the TMM, the band structure of an infinite periodic system can be calculated, but the main advantage of this method is the calculation of transmission and reflection properties of EM waves of various frequencies incident on a finite thickness slab of PBG material. The inset in Fig. 2(a) shows the transmission from four-layer st type of crystal (solid line), which agrees well with the simulation results (dotted line).

It was previously shown in other metallic layer-by-layer photonic crystals that defect structures around this geometry can be built by means of adding or removing rods from an otherwise perfect crystal. ${ }^{9}$ The same idea was used to investigate the defect characteristics of these semiconductor based photonic crystals. Figure 3(a) shows the transmission through a nine-layer fct type of crystal, with a single rod missing from the fifth layer of the crystal. The resonance frequency of the defect mode is at $91.8 \mathrm{GHz}$, with a $Q$ factor (quality factor), defined as the center frequency divided by the full width at half maximum, of 35 .

We then investigated a planar type of defect structure, built around an eight-layer fct based photonic crystal. The planar defect was obtained by separating the fourth and fifth layers of the crystal. This resulted in a planar air gap between the two photonic mirrors, each formed of a four layer crystal. 


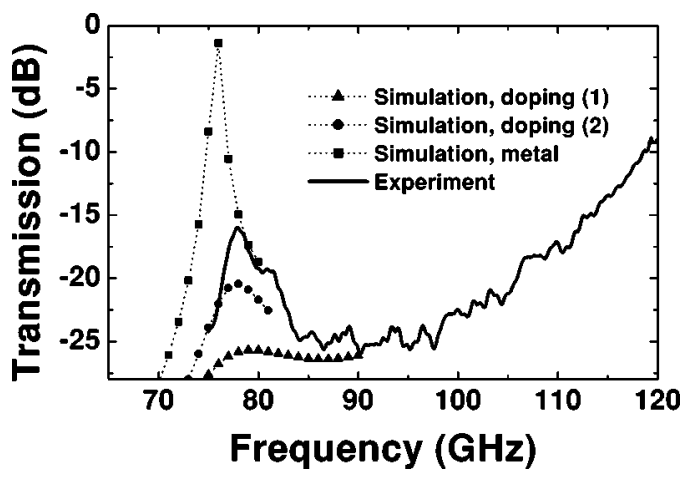

FIG. 4. Calculated transmission spectra of a planar defect for two different doping concentrations (circles and triangles), for a complete metallic structure (squares) and the experimental result of the same defect structure (solid line).

These planar defects also resulted in similar defect characteristics, however with higher transmission amplitudes. Figure 3(b) shows the transmission through a planar defect with separation width $L=550 \mu \mathrm{m}$ (solid line). The resonance frequency of the defect mode is at $90 \mathrm{GHz}$ with a $Q$ factor of 25 . As the separation of the cavity was increased to $L$ $=650 \mu \mathrm{m}$, the resonance frequency of the defect mode shifted to $83 \mathrm{GHz}$ (dotted line). These results indicate that the defect frequency can be tuned within the band gap by changing the width of the cavity. ${ }^{9}$

In order to understand the effect of doping concentration on defect characteristics, we compared our experimental results with the TMM simulations (Fig. 4). We used the same planar defect structure described above, with a separation width of $800 \mu \mathrm{m}$. The results of the simulations for a doping concentration of $10^{17} \mathrm{~cm}^{-3}$, which corresponds to resistivity of $0.09 \Omega \mathrm{cm}$, is shown by triangles. As the doping concentration is increased to $10^{19} \mathrm{~cm}^{-3}$ (resistivity of $0.006 \Omega \mathrm{cm}$ ), the simulation results showed a significant increase in the $Q$ factor and the transmission amplitude of the defect mode (Fig. 4, circles). Having lower resistivity, the measured transmission amplitude and $Q$ factor for our samples are higher (Fig. 4, solid line). The simulation results of a complete metallic structure, which is shown by squares, indicate that our results may still be improved to reach better defect characteristics using wafers with higher doping concentrations.

In conclusion, by using standard semiconductor micromachining techniques, we fabricated layer-by-layer quasimetallic photonic crystals. These crystals exhibited a metallicity gap with an upper band edge around $100 \mathrm{GHz}$. The rejection rate per layer obtained from fct type crystals (5.3 $\mathrm{dB}$ ) was found to be superior to the rejection rates of similar dielectric photonic crystal structures $(3.5-4 \mathrm{~dB}$ per layer at midgap frequencies). Cavities created by removing rods resulted in localized defect modes at certain resonant frequencies. We observed the tunability of this resonant frequency for planar type defects. We showed that the doping concentration of the wafers is an important factor in determining the defect characteristics, and proposed that photonic crystals fabricated with wafers having higher doping concentrations will have higher $Q$ factors and higher transmission amplitudes.

This work was supported by NATO Grant No. SfP971970, by National Science Foundation Grant No. INT9820646, and by Turkish Defense Ministry Grant No. KOBRA-01, and by Thales JP8.04. Ames Laboratory is operated for the U.S. Department of Energy by Iowa State University under Contract No. W-7405-Eng-82.

${ }^{1}$ J. D. Joannopoulos, R. D. Meade, and J. N. Winn, Photonic Crystals: Molding the Flow of Light (Princeton University Press, Princeton, NJ, 1995).

${ }^{2}$ For a recent review, see, for example, articles in Photonic Band Gap Materials, edited by C. M. Soukoulis (Kluwer, Dortrecht, 1996).

${ }^{3}$ K. M. Ho, C. T. Chan, C. M. Soukoulis, R. Biswas, and M. Sigalas, Solid State Commun. 89, 413 (1994).

${ }^{4}$ E. Ozbay, J. Opt. Soc. Am. B 13, 1945 (1996).

${ }^{5}$ D. R. Smith, S. Schultz, N. Kroll, M. M. Sigalas, K. M. Ho, and C. M. Soukoulis, Appl. Phys. Lett. 65, 645 (1994).

${ }^{6}$ E. R. Brown and O. B. McMahon, Appl. Phys. Lett. 67, 2138 (1995).

${ }^{7}$ D. F. Sievenpiper, M. E. Sickmiller, and E. Yablonovitch, Phys. Rev. Lett. 76, 2480 (1996).

${ }^{8}$ J. B. Pendry, A. J. Holden, W. J. Stewart, and I. Youngs, Phys. Rev. Lett. 76, 4773 (1996).

${ }^{9}$ B. Temelkuran, H. Altug, and E. Ozbay, IEE Proc.-J: Optoelectron. 145, 409 (1998).

${ }^{10}$ J. B. Pendry, A. J. Holden, D. J. Robbins, and W. J. Stewart, IEEE Trans. Microwave Theory Tech. 47, 2075 (1999); J. Phys.: Condens. Matter 10, 4785 (1998).

${ }^{11}$ F. Gadot, A. de Lustrac, J.-M. Lourtioz, T. Brillat, A. Ammouche, and E. Akmansoy, J. Appl. Phys. 85, 8499 (1999).

${ }^{12}$ E. Ozbay, A. Abeyta, G. Tuttle, M. Tringides, R. Biswas, C. T. Chan, C. M. Soukoulis, and K. M. Ho, Phys. Rev. B 50, 1945 (1994).

${ }^{13}$ E. Ozbay, E. Michel, G. Tuttle, R. Biswas, M. M. Sigalas, and K. M. Ho, Appl. Phys. Lett. 64, 2059 (1994).

${ }^{14}$ J. G. Fleming and S. Y. Lin, Opt. Lett. 24, 49 (1999).

${ }^{15}$ S. Noda, N. Yamamoto, H. Kobayashi, M. Okano, and K. Tomoda, Appl. Phys. Lett. 75, 905 (1999).

${ }^{16}$ D. F. Sievenpiper, E. Yablonovitch, J. N. Winn, S. Fan, P. R. Villeneuve, and J. D. Joannopoulos, Phys. Rev. Lett. 80, 2829 (1998).

${ }^{17}$ S. Gupta, G. Tuttle, M. M. Sigalas, and K. M. Ho, Appl. Phys. Lett. 71, 2412 (1997).

${ }^{18}$ K. A. McIntosh, L. J. Mahoney, K. M. Molvar, O. B. McMahon, S. Verghese, M. Rothschild, and E. R. Brown, Appl. Phys. Lett. 70, 2937 (1997).

${ }^{19}$ J. B. Pendry and A. MacKinnon, Phys. Rev. Lett. 69, 2772 (1992).

${ }^{20}$ M. M. Sigalas, C. M. Soukoulis, E. N. Economou, C. T. Chan, and K. M. Ho, Phys. Rev. B 48, 14121 (1993). 